\title{
FUZZY RANDOM KOROVKIN THEORY AND INEQUALITIES
}

\author{
George A. AnASTASSIOU
}

\begin{abstract}
We introduce and study the fuzzy random positive linear operators acting on fuzzy random continuous functions. We establish a series of fuzzy random Shisha-Mond type inequalities of $L^{q}$-type $1 \leqslant q<\infty$ and related fuzzy random Korovkin type theorems, regarding the fuzzy random $q$-mean convergence of fuzzy random positive linear operators to the fuzzy random unit operator for various cases. All convergences are with rates and are given via the above fuzzy random inequalities involving the fuzzy random modulus of continuity of the engaged fuzzy random function. The assumptions for the Korovkin theorems are minimal and of natural realization, fulfilled by almost all example fuzzy random positive linear operators. The astonishing fact is that the real Korovkin test functions assumptions are enough for the conclusions of our fuzzy random Korovkin theory. We give at the end applications.
\end{abstract} $60 \mathrm{H} 99$.

Mathematics subject classification (2000): 26E50, 28E10, 41A17, 41A25, 41A36, 47S40, 60H25,

Key words and phrases: fuzzy random positive linear operator, fuzzy random Korovkin theory and inequalities, fuzzy random Shisha-Mond inequality, fuzzy random modulus of continuity, fuzzy stochastic process, fuzzy random function.

\section{REFERENCES}

[1] G. A. Anastassiou, Moments in Probability and Approximation Theory, Pitman/Longman, \#287, UK, 1993.

[2] G. A. ANASTASSIOU, Rate of convergence of fuzzy neural network operators, univariate case, Journal of Fuzzy Mathematics, 10, (3) (2002), 755-780.

[3] G. A. AnASTASSIOU, Univariate fuzzy-random neural network approximation operators, Computers and Mathematics with Applications, 48, (2004), 1263-1283.

[4] G. A. Anastassiou, On basic fuzzy Korovkin theory, Studia Univ."Babes-Bolyai" Mathematica, L, (4) Dec.2005, 3-10.

[5] G. A. Anastassiou, Fuzzy Korovkin theorems and inequalities, The Journal of Fuzzy Mathematics, 14, (3) (2006).

[6] G. A. Anastassiou, On fuzzy global smoothness preservation, The Journal of Fuzzy Mathematics, 14, (3) 2006.

[7] G. ANASTASSIOU, S. GAL, On a fuzzy trigonometric approximation theorem of Weierstrass-type, Journal of Fuzzy Mathematics, Los Angeles, 9, (3) (2001), 701-708.

[8] G. Anastassiou, S. GaL, On fuzzy trigonometric Korovkin theory, J.Nonlinear Functional Analysis and Appl, (2005).

[9] D. BUtNARIU, Measurability concepts of fuzzy mappings, Fuzzy Sets and Systems, 31, (1989), 77-82.

[10] R. DudLey, Real Analysis and Probability, Wadsworth \& Brooks/Cole Mathematics Series, Pacific Grove, CA, 1989.

[11] S. GaL, Approximation theory in Fuzzy setting, Chapter 13, 617-666, in Handbook of AnalyticComputational Methods in Applied Mathematics, editor, G.A. Anastassiou, Chapman \& Hall/CRC, Boca Raton, 2000.

[12] R. GoETSCHEL JR., W. VoXman, Elementary fuzzy calculus, Fuzzy Sets and Systems, 18, (1986), 31-43.

[13] O. KaleVA, Fuzzy differential equations, Fuzzy Sets and Systems, 24, (1987), 301-317. 
[14] YUn KyONG KIM, ByUng MoOn GHIL, Integrals of fuzzy-number-valued functions, Fuzzy Sets and Systems, 86, (1997), 213-222.

[15] P. P. Konovkin, Linear Operators and Approximation Theory, Hindustan Publ. Corp., Delhi, India, 1960.

[16] O. SHISHA, B. MOND, The degree of convergence of sequences of linear positive operators, Nat. Acad. of Sci. U.S., 60, (1968), 1196-1200.

[17] Congxin Wu, Ming Ma, On embedding problem of fuzzy number space: Part 1, Fuzzy Sets and Systems, 44, (1991), 33-38.

[18] CongXIN WU, ZENGTAI Gong, On Henstock integral of fuzzy-number-valued functions (I), Fuzzy Sets and Systems, 120, (2001), 523-532. 